\title{
Associations between pre-pregnancy body mass index, gestational weight gain and preterm birth: a cohort study in Wuhan, China
}

\section{Yiyang Guo}

Huazhong University of Science and Technology Tongji Medical College School of Public Health

Chao Xiong

Wuhan Women and Children Medical Care Center

Aifen Zhou

Wuhan Women and Children Medical Care Center

Ronghua Hu

Wuhan Women and Children Medical Care Center

\section{Rong Yang}

Wuhan Women and Children Medical Care Center

Yukai Du ( $\nabla$ duyukai10487@sina.com )

Huazhong University of Science and Technology Tongji Medical College School of Public Health https://orcid.org/0000-0003-4717-2603

\section{Research}

Keywords: Body mass index, China, Gestational weight gain, Preterm birth

Posted Date: October 19th, 2020

DOl: https://doi.org/10.21203/rs.3.rs-49045/v2

License: (9) This work is licensed under a Creative Commons Attribution 4.0 International License. Read Full License 


\section{Abstract}

Background Preterm birth (PTB) is the leading cause of neonatal mortality and morbidity worldwide.

Methods This cohort study aims to investigate the associations between pre-pregnancy BMI, total gestational weight gain (GWG), and the GWG during early pregnancy with PTB utilizing data of 83,096 Chinese women from the Wuhan Maternal and Children Healthcare Information Tracking System in China.

Results Women who were underweight, overweight or obese before pregnancy had an higher overall risk of PTB compared with the normal group. Women who with a total GWG below the IOM recommendation had an increased risk of PTB compared to women who had GWG within the recommendation, whereas increased weekly early pregnancy GWG had a significant impact on the increasing risk of PTB. When stratified by subtypes of PTB, pre-pregnancy underweight was associated with higher risk of spontaneous PTB, and pre-pregnancy overweight /obese increased the risk of both spontaneous PTB and medically indicated PTB. Women with total GWG below the IOM recommendation had elevated risk for spontaneous PTB and PROM, and women with GWG above the recommendation had decreased risk for all three subtypes of PTB, whereas risk for the three subtypes of PTB increased along with increasing weekly GWG of early pregnancy.

Conclusions Maternal underweight, overweight/obesity, total GWG, and GWG during early pregnancy should be considered in combination to reduce the risk of PTB, women should modify their weight gains during pregnancy according to the results.

\section{Background}

Preterm birth (PTB), defined as a delivery of live born infant before 37 completed gestational weeks[1], is the leading cause of neonatal mortality and morbidity worldwide[2], and has been reported to be strongly associated with long-term health problems such as neurological disabilities and various chronic diseases[3, 4]. During the recent decades, the burden of preterm birth is substantial and increasing[5]. Therefore, it is important to identify the potential modifiable risk factors for prevention of preterm birth. However, as a complex phenomenon, the etiology of preterm birth is not yet well understood to date.

Several previous studies have indicated that the maternal overweight/obesity is one potential modifiable risk factor for PTB $[6,7]$, and thus provided a target for intervention of PTB during pre-conception care. Besides, as weight control is considered to be more feasible during pregnancy than before conception, much attention has been drawn to the association of gestational weight gain (GWG) with PTB in recent years. However, conclusions of previous investigations have been inconsistent, as several studies reported an association between lower GWG and elevated risk of PTB[8], while some studies indicated that risk of PTB increased with higher GWG[9]. 
Most of the previous studies only evaluated the GWG by weight data throughout pregnancy, which may lead to biased associations because GWG differs by term and preterm birth[10], and is not linear throughout pregnancy[11]. GWG during early pregnancy was considered to be critical for embryogenesis and fetal growth[12], however, few studies have specifically examined GWG early in pregnancy related to PTB. Furthermore, PTB is a heterogeneous condition, but few studies have examined whether associations between pre-pregnancy BMI, GWG and PTB differ by different subtypes of PTB. Besides, most of previous studies were conducted in developed countries. Commonly, above $30 \%$ of reproductive age women generally have a high body mass index $\left(\mathrm{BMl} \geq 23.0 \mathrm{~kg} / \mathrm{m}^{2}\right)$ in western United States[13], however囚this number in developing countries was only $8.5 \%{ }^{14}$, and was less investigated.

Therefore, the magnitude and direction of the associations between pre-pregnancy BMI, GWG and PTB has not been well studied, especially in developing countries. Hence, a retrospective cohort study with the records of 83,096 women was conducted in China to investigate the independent as well as joint association of pre-pregnancy BMI, total GWG and early pregnancy GWG with the risk of subtypes of PTB in singleton pregnancies.

\section{Methods}

\section{Study Population}

This retrospective cohort study was conducted in Wuhan, China, using electronic medical record (EMR) data from the Wuhan Maternal and Children Healthcare Information Tracking System, which is a large integrated healthcare system including the information of maternal demographic characteristics, past histories, antenatal examinations and delivery information from 93 hospitals with obstetrics and gynecology departments and 121 primary health institutions in Wuhan. Eligible participants in this study should met the following criteria: (1) women who delivered a live singleton newborn without any birth defect within 28-41 weeks' gestational age between June 1, 2015 and June 1, 2017; (2) lived in the urban area of Wuhan during the whole pregnancy and (3), at least had two weight records during early pregnancy, once earlier than 9 weeks of gestation and another should not later than 20weeks of gestation. Women who were younger than 16 years or older than 50 years at delivery were excluded. Also, participants with unknown anthropometric data (i.e. maternal height, pre-pregnancy weight and weight at delivery) were excluded.

A total of 110,078 electronic medical records were conducted and 83,096 participants met the eligibility criteria and were included in the study. 68,527 of them had at least two weight measurement records during 8-20 gestational weeks.

\section{Assessment of study variables}

Gestational age was calculated from the delivery date and the date of the last recorded normal menstrual period. Preterm delivery was defined as a delivery between 28 weeks 0 days and 36 weeks 6 days of gestation. We excluded very preterm deliveries ( $<28$ weeks gestation) as there were few in this cohort. 
We additionally categorized preterm term subtype as either spontaneous preterm birth, premature rupture of membranes (PROM), or medically indicated preterm birth, based on the records of clinical diagnosis reported by the obstetrician at birth. Medically indicated preterm birth was defined by either induction or caesarean section without uterine contractions or rupture of membranes prior to delivery. PROM was defined as birth with premature rupture of membranes, and spontaneous preterm birth was identified as early onset of delivery and no identifiable medical indication, without a PROM diagnosis.

Gender of the infants was obtained from birth records. Pre-pregnancy weight and height were selfreported at the first prenatal check up (usually in the first trimester). BMI before pregnancy was calculated by weight $(\mathrm{kg}) /$ height $\left(\mathrm{m}^{2}\right)$ and then divided into four groups according to the recommendations of the Institute of Medicine (IOM) (2009): (1) underweight (<18.5 kg/m²); (2) normal weight $\left(18.5-24.9 \mathrm{~kg} / \mathrm{m}^{2}\right)$; (3) overweight (25.0-29.9 kg/m²); and (4) obese ( $\left.\geq 30 \mathrm{~kg} / \mathrm{m}^{2}\right)[11]$.

Maternal weight at delivery was measured within 3 days before the delivery day, and the calculation of GWG was to subtract the maternal pre-pregnancy weight from the weight at delivery, and then classified according to the recommendations of the Institute of Medicine (IOM) (2009). GWG within the IOM recommendations was defined as $12.5-18 \mathrm{~kg}, 11.5-16 \mathrm{~kg}, 7-11.5 \mathrm{~kg}$, and 5-9 $\mathrm{kg}$ respectively for underweight, normal weight, overweight, and obese women.

According to evidence from previous studies, the gestational BMI gain was divided into minimal $(<5$ $\left.\mathrm{kg} / \mathrm{m}^{2}\right)$, moderate $\left(5-10 \mathrm{~kg} / \mathrm{m}^{2}\right)$ and excessive $\left(>10 \mathrm{~kg} / \mathrm{m}^{2}\right)$ [13]. Every increase in BMI by 1 point is roughly equivalent to $2.5 \mathrm{~kg}$ in weight gain, using the average female weight and height at reproductive age $(158 \mathrm{~cm}, 54 \mathrm{~kg})$ in China [14].

We used average weekly weight gain between 8-20 weeks gestation to evaluate GWG during early pregnancy, which was calculated as the latest prenatal care weight before 20 weeks of gestation minus the first prenatal care weight(before 9 weeks) divided by gestation age of the latest prenatal care (up to 20 weeks of gestation) and classified as class I ( $<200 \mathrm{~g} /$ week), class II (200-400 g/week), class III (400-600 $\mathrm{g} /$ week), and class IV (> $600 \mathrm{~g} /$ week). [15]

\section{Statistical Analysis}

Unconditional logistic regression was used to calculate odds ratios (ORs), and 95\% confidence intervals (Cls) to evaluate the association between PTB and pre-pregnancy BMI, GWG and gestational BMI gain. Models were adjusted for some potential confounders, including infant gender, birth weight, maternal age, parity囚education level and models that evaluated maternal pre-pregnancy BMI and BMI/weight gain during pregnancy were mutually adjusted.

Analyses were further stratified by maternal pre-pregnancy BMI categories, and effect modifications with these variables were evaluated by including the relevant cross-product terms in the regression models. Linear trends were tested using the Wald test. Statistical analyses were conducted with SAS, version 9.4, (SAS Institute, Inc., Cary, North Carolina) and P values $<0.05$ was considered statistically significant. 


\section{Results}

Table 1 lists the selected characteristics of the participants in the cohort. 3,983 out of $83,096(4.79 \%)$ women delivered a preterm birth infant. Among all the preterm births, 1,569 (39.39\%) were spontaneous preterm births, 1,337 (33.57\%) were premature rupture of membranes and 1,077 (27.04\%) were medically indicated preterm births. Women over the age of 30 , multiparous women, women who gave birth to a male infant, and were overweight or obese before pregnancy were more likely to have preterm birth. The mean total GWG among women who had preterm birth was $14.87 \pm 5.99 \mathrm{~kg}$, lower than that of women with term birth $(17.57 \pm 6.94 \mathrm{~kg})$.

Table1. Distribution of selected characteristics among full term births and preterm births 


\begin{tabular}{|c|c|c|c|c|c|}
\hline Maternal characteristic & $\begin{array}{l}\text { Full term births } \\
(\mathrm{n}=79,113)\end{array}$ & Preterm births $(\mathrm{n}=3,983)$ & \multirow[b]{2}{*}{$\%$} & & \\
\hline $\mathrm{n}$ & $\%$ & $\mathrm{n}$ & & & \\
\hline \multicolumn{6}{|l|}{ Age at delivery (years) } \\
\hline$<25$ & 14897 & 96.05 & 613 & 3.95 & \\
\hline $25-29$ & 41044 & 95.78 & 1809 & 4.22 & \\
\hline $30-34$ & 18135 & 94.10 & 1138 & 5.90 & \\
\hline$\geq 35$ & 5037 & 92.25 & 423 & 7.75 & \\
\hline \multicolumn{6}{|l|}{ Education Level } \\
\hline Less than high school & 9043 & 95.48 & 428 & 4.52 & \\
\hline High school & 35587 & 95.10 & 1834 & 4.90 & \\
\hline College & 30533 & 95.25 & 1522 & 4.75 & \\
\hline Advanced Degree & 3950 & 95.20 & 199 & 4.80 & \\
\hline \multicolumn{6}{|l|}{ Gravidity } \\
\hline 1 & 40853 & 95.12 & 2097 & 4.88 & \\
\hline 2 & 20448 & 95.63 & 934 & 4.37 & \\
\hline$\geq 3$ & 17812 & 94.93 & 952 & 5.07 & \\
\hline \multicolumn{6}{|l|}{ Parity } \\
\hline Nulliparous & 65981 & 95.33 & & 3231 & 4.67 \\
\hline Multiparous & 13132 & 94.58 & & 752 & 5.42 \\
\hline \multicolumn{6}{|l|}{ Offspring Gender } \\
\hline Male & 41859 & 94.56 & 2406 & 5.44 & \\
\hline Female & 37254 & 95.94 & 1577 & 4.06 & \\
\hline \multicolumn{6}{|c|}{ Prepregnancy BMI $\left(\mathrm{kg} / \mathrm{m}^{2}\right)$} \\
\hline Under weight (<18.5) & 13453 & 95.32 & 661 & 4.68 & \\
\hline
\end{tabular}




\begin{tabular}{|lllll|} 
Normal (18.5-24.9) & 60755 & 95.33 & 2973 & 4.67 \\
\hline Overweight (25-29.9) & 4365 & 93.43 & 307 & 6.57 \\
\hline Obese $(\geq 30)$ & 540 & 92.78 & 42 & 7.22 \\
\hline
\end{tabular}

Table 2 shows the relationships between pre-pregnancy BMI, gestational BMI gain, total GWG, GWG during early pregnancy and PTB. Compared with women of normal pre-pregnancy BMI, women who were underweight [Adjusted OR:1.05 (1.03-1.27)], overweight[Adjusted OR:1.28 (1.12-1.43)] or obese [Adjusted OR:1.50 (1.11-1.98)] prior to pregnancy had an raised risk of PTB. Compared with women who had a total GWG within the IOM recommendation, women with a total GWG below the recommendation had an increased risk of PTB[Adjusted OR:1.11 (1.02-1.21)], while those with a total GWG above the recommendation had a lower risk of PTB [Adjusted OR:0.45 (0.42-0.49)].Further, decreased risk of PTB was observed for women with greater BMI gain during pregnancy ( $\mathrm{f}$ for trend $<0.01$ ). The adjusted OR for women who had a gestational BMI gain more than $10 \mathrm{~kg} / \mathrm{m}^{2}$ was $0.29(\mathrm{Cl}: 0.25-0.33)$ compared with women who gained less than $5 \mathrm{~kg} / \mathrm{m}^{2}$ during pregnancy.

The association between early pregnancy GWG and PTB in the first trimester shown different trends. As average GWG increased during early pregnancy, an elevated risk of PTB was observed ( $p$ for trend $<0.01$ ). Compared with women who gained less than 200 grams per week before 20 weeks of pregnancy, women who gained greater than 400 grams per week had a significantly higher risk of PTB [Adjusted OR:1.50 (1.33-1.69)]. It was worth noting that women who gained more than 600 grams per week at the beginning of pregnancy had the highest risk of PTB, and the adjusted OR was 2.23 (Cl: 2.01-2.48).

Table 2. Associations of pre-pregnancy BMI, gestational BMI gain, total GWG, and GWG during early pregnancy with risk of PTB ${ }^{a}$ 


\begin{tabular}{|c|c|c|c|c|}
\hline Variables & $\begin{array}{l}\text { Full term } \\
\text { births (n) }\end{array}$ & $\begin{array}{l}\text { Preterm } \\
\text { births (n) }\end{array}$ & $\begin{array}{l}\text { Crude } \\
\text { OR(95\% Cl) }\end{array}$ & $\begin{array}{l}\text { Adjusted } \\
\text { OR(95\% } \mathrm{Cl})^{\star}\end{array}$ \\
\hline \multicolumn{5}{|l|}{ Pre-pregnancy BMI $\left(\mathrm{kg} / \mathrm{m}^{2}\right)^{b}$} \\
\hline Underweight (<18.5) & 13453 & 661 & $\begin{array}{l}1.02(0.93- \\
1.13)\end{array}$ & $1.05(1.03-1.27)$ \\
\hline Normal (18.5-24.9) & 60755 & 2973 & 1.00 (ref) & 1.00 (ref) \\
\hline Overweight (25-27.9) & 4365 & 307 & $\begin{array}{l}1.51(1.36- \\
1.71)\end{array}$ & $1.28(1.12-1.43)$ \\
\hline Obese $(\geq 30)$ & 540 & 42 & $\begin{array}{l}1.87(1.41- \\
2.45)\end{array}$ & $1.50(1.11-1.98)$ \\
\hline \multicolumn{5}{|l|}{$\begin{array}{l}\text { Total GWG By IOM } \\
\text { Recommendation }^{b}\end{array}$} \\
\hline Below & 12975 & 977 & $\begin{array}{l}1.07(0.99- \\
1.17)\end{array}$ & $1.11(1.02-1.21)$ \\
\hline Within & 23365 & 1640 & 1.00 (ref) & 1.00 (ref) \\
\hline Above & 42773 & 1366 & $\begin{array}{l}0.46(0.42- \\
0.49)\end{array}$ & $0.45(0.42-0.49)$ \\
\hline
\end{tabular}

\section{Gestational BMI gain $\left(\mathrm{kg} / \mathrm{m}^{2}\right)$

$$
\text { b }
$$

\begin{tabular}{lllll}
$<5$ & 18875 & 1468 & $1.00($ ref $)$ & 1.00 (ref) \\
\hline $5-10$ & 51452 & 2322 & $\begin{array}{l}0.58(0.54- \\
0.62)\end{array}$ & $0.59(0.55-0.63)$ \\
& & & $0.28(0.24-$ & $0.29(0.25-0.33)$ \\
\hline 10 & 8786 & 193 & $0.33)$ &
\end{tabular}

$P$ for trend $\quad<0.01$

Average GWG up to 20

weeks (g/week) ${ }^{c}$

\begin{tabular}{|c|c|c|c|c|}
\hline$<200$ & 38408 & 1489 & 1.00 (ref) & 1.00 (ref) \\
\hline $200-399$ & 15048 & 627 & $\begin{array}{l}1.08(0.98- \\
1.18)\end{array}$ & $1.07(0.97-1.18)$ \\
\hline $400-599$ & 6128 & 359 & $\begin{array}{l}1.51(1.34- \\
1.70)\end{array}$ & $1.50(1.33-1.69)$ \\
\hline$\geq 600$ & 5950 & 518 & $\begin{array}{l}2.25(2.03- \\
2.49)\end{array}$ & $2.23(2.01-2.48)$ \\
\hline
\end{tabular}

$P$ for trend $<0.01$ 
1. Gestational BMI gain, total GWG, and GWG during early pregnancy were evaluated in separate models.

2. Adjusted for maternal age at delivery, education level, gravidity, parity, parityand offspring sex. Additionally, pre-pregnancy BMI, and gestational BMI gain were mutually adjusted. Total GWG also adjusted for pre-pregnancy BMI. $(n=83,096)$

3. Adjusted for maternal age at delivery, education level, gravidity, parity, offspring sex, and prepregnancy BMI. $(n=68,527)$

Results for the associations of evaluated variables with subtypes of PTBare presented in Table 3 (See additional file 1). After adjustment for potential confounders, pre-pregnancy underweight was associated with higher odds of spontaneous preterm birth [Adjusted OR:1.33 $(1.17,1.52)]$, and pre-pregnancy overweight and obese increased the risk of both spontaneous preterm birth [Adjusted OR for overweight: $1.32(1.10,1.59)$; obese:1.68 $(1.10,2.56)$ ] and medically indicated preterm birth [Adjusted OR for overweight: $1.63(1.33,2.00)$; obese: Adjusted OR $1.77(1.07,2.93)]$. Women with total GWG below the IOM recommendation had elevated risk for spontaneous preterm birth [Adjusted OR: $1.13(1.00,1.28)$ ] and premature rupture of membrane [Adjusted OR: $1.18(1.02,1.36)$ ], and women with GWG above the recommendation had decreased risk for all the subtypes of preterm birth. Adjusted odds for three subtypes of preterm birth decreased as gestational BMI gain increased ( $p$ for trend $<0.01$ ), whereas the risk for three subtypes of preterm birth increased along with increasing weekly GWG of early pregnancy ( $p$ for trend $<0.01$ ).

In addition, we examined the relationship between PTB stratified by pre-pregnancy BMI and the gestational BMI gain and GWG in the early pregnancy (See additional file 2, Table 4). Women with increased BMI during the entire pregnancy had a significantly lower risk of PTB in all pre-pregnancy BMI categories ( $p$ for trend $<0.01$ ). In contrast, among women who were underweight/normal weight before pregnancy, as the average GWG during early pregnancy increased, the risk of PTB was observed to increase ( $p$ for trend $<0.01$ ). In particular, women who were underweight before pregnancy and had the highest average GWG during the early pregnancy ( $\geq 600 \mathrm{~g} /$ week) had the highest risk of PTB[Adjusted OR:4.61 (3.68-5.77)], whereas the corresponding ORs were still elevated but not so strong in women who had normal weight before pregnancy [Adjusted OR:1.88 (1.66-2.14)]. However, no significantly association of the risk of PTB and GWG during early pregnancy was observed among women who were overweight/obese before pregnancy ( $p$ for trend $=0.79$ ). There was significant heterogeneity between the pre-pregnancy BMI categories and the association between GWG during early pregnancy and PTB risk ( $P$ for heterogeneity<0.01).

\section{Discussion}

In this cohort study of Chinese women, we found that pre-pregnancy overweight/obesity was independently associated with an increased overall risk of preterm birth, which is consistent with previous findings $[6,7]$. When stratified by subtypes, pre-pregnancy overweight/obesity was observed to 
significantly increase the risk of medically indicated preterm birth and spontaneous preterm birth in our study, but not the preterm caused by premature rupture of membrane.

The mechanisms linking pre-pregnancy overweight/obesity with risk of PTB is not well understood to date, but probably involves inflammatory, neuroendocrine and lifestyle factors[15]. Goldenberg and Culhane[16] have indicated that preterm birth is mediated by increased systemic inflammation due to a wide range of pre-pregnancy risk factors. Gestational diabetes, pre-eclampsia and obesity are also related to increased systemic inflammation, sometimes called the metabolic syndrome of pregnancy. Especially central obesity, which is more strongly related to insulin resistance than obesity per se, predisposes individuals to these diseases.[16, 17], which are known contributors to medically indicated preterm birth. Another previous study also suggested that the association between maternal overweight /obesity and excess risk of medically indicated preterm birth may largely be due to obesity-related pregnancy disorders[18].

On the other end of the pre-pregnancy BMI spectrum, pre-pregnancy underweight has been reported to be associated with an increased risk of PTB $[8,19]$, though fewer studies have separated different subtypes of preterm birth. In this study, we found that women who were underweight before pregnancy were at greater risk of overall PTB. However, results for subtypes of PTB showed that pre-pregnancy underweight was associated with higher risk of spontaneous PTB, while no significant association of medically indicated PTB with pre-pregnancy underweight was found in our study. This result is consistent with a previous study from Boston Birth Cohort[20].

In contrast to the more consistent evidence linking pre-pregnancy BMI with PTB, studies for association of gestational weight gain (GWG) and PTB have yielded inconsistent results. Although some previous studies have shown an association between lower GWG and increased risk of preterm birth[8], some studies indicated a positive association between excessive GWG and elevated risk of preterm birth[9]. In our study, it was found that low GWG (below the IOM recommendation) throughout pregnancy had significant impact on the increased risk of Spontaneous PTB and PROM, while excessive GWG (above IOM recommendation) was found to be associated with decreased risk for all types of PTB. Since some studies believed that BMI is more representative of body fat than body weight alone[13], we also classified weight gain during pregnancy based on the net change in BMI. Similarly, excessively increase in BMI throughout pregnancy was also related to a decreased risk of all types of PTB. Rebecca et al. found a similar conclusion in a systemic review and meta-analysis of 1.3 million pregnancies that gestation weight gain above recommendations was associated with lower risk of preterm birth[21]. According to a recent systematic review by McDonald et al[22]., women with high total GWG were observed to have lower risks of PTB, high weekly GWG was associated with increased PTB. This observation points to the need more study, although the effect may be due to the association of conditions such as preeclampsia, which is often accompanied by edema and significant short-term increased in weight.

However, most of the previous studies evaluating the association of GWG with PTB relied on only two weight measures: weight near conception and weight at delivery, which may have biased associations 
because GWG over gestational periods differs by term and preterm birth[10]. Furthermore, GWG during early pregnancy was considered to be critical for embryogenesis and fetal growth[12], however, few studies have specifically examined GWG early in pregnancy related to PTB. Therefore, in this study, we also investigated the association of average GWG during early pregnancy and PTB, and our results indicated that high weekly GWG of early pregnancy was significantly associated with higher risk of all types of PTB. While GWG in the first half of pregnancy is mainly the result of maternal tissues deposition and placental growth, gains from that point on until the end of pregnancy might be influenced by accumulation of amniotic fluid. As high GWG is associated with inflammatory up-regulation through increased production of adipokines by adipose tissue and augmented systemic secretion of proinflammatory cytokines, which may contribute to the biological pathway of PTB.

To explore whether the pre-pregnancy BMI would modify the association between GWG and PTB risk, we stratified the association according to pre-pregnancy BMI categories. We found that there was a significant correlation between the excessive increase of BMI throughout pregnancy and the reduction in PTB risk for all pre-pregnancy BMI categories, whereas the high weekly GWG during the early pregnancy is associated with a significantly elevated risk of PTB among women with underweight or normal weight in the early pregnancy. Interestingly, for women who were underweight before pregnancy, the association between high weekly GWG of early pregnancy and PTB was strongest. In contrast to our results, findings of Sharma et al. do not support any significant association between GWG in the first and second trimester and PTB among underweight and normal weight women[10]. Further prospective studies are needed to examine whether a causative relationship between pre-pregnancy BMI, GWG of different periods of pregnancy, and PTB exists, as well as the biological mechanisms underlying this relationship.

One of the strengths of this study is a large population-based cohort study which allowed us to evaluate the role of both total GWG and early GWG in PTB risk, because the anthropometric characteristics of participants during early pregnancy could be obtained easily. Also, we were able to identity subtypes of PTB in this study. To our knowledge, this is the first study investigating the association between pre-pregnancy BMI, total GWG as well as early pregnancy GWG and different subtypes of PTB among Chinese women. The potential limitation of the study is that our data relies on a self-reported pre-pregnancy weight, which may be underestimated and may have potential classification bias. However, previous researches has shown that BMI categories based on self-reported data rarely change, and it could be considered that self-reported weight could replace actual measurements[23, 24].

\section{Conclusion}

A population-based cohort study was conducted in China to explore the relationship between prepregnancy BMI, total GWG, early pregnancy GWG and the risk of PTB. We found that pre-pregnancy overweight and obesity were independently associated with the greater risk of medically indicated preterm birth and spontaneous preterm birth, whereas pre-pregnancy underweight was associated with higher risk of spontaneous PTB. High BMI gain during the whole pregnancy was shown to be related to a decreased risk of all types of PTB. In contrast, high weekly GWG of early pregnancy was significantly 
associated with higher risk of all types of PTB. Our results indicate that maternal underweight, overweight/obesity, total GWG, and GWG during early pregnancy should be considered in combination to reduce the risk of PTB.Further prospective studies are needed to examine the relationship of trimesterspecific GWG and PTB, as well as the underlying biological mechanisms.

\section{Declarations}

\section{Ethics approval and consent to participate}

The study was approved by the Institutional Review Board (IRB) of the School of Public Health, Tongji Medical College, Huazhong University of Science and Technology. And all methods were carried out according to the approved guidelines established by the IRB. Informed consent was obtained from all the participants when their EMR data was being typed into computers.

\section{Consent for publication}

Not applicable

\section{Availability of data and materials}

The datasets generated and/or analysed during the current study are available in the [Open Science Framework] repository, [https://osf.io/vsprj/]

DOI 10.17605/OSF.IO/VSPRJ

\section{Competing interests}

There are no conflicts of interest among the authors.

\section{Funding}

There was no funding in this research.

\section{Author Contribution}

The first author Yiyang Guo contributed to the study design, data analysis, and manuscript preparation. Chao Xiong and Aifen Zhou contributed to the data analysis and manuscript revision. Ronghua Hu and Rong Yang revised the manuscript. The corresponding authors Yukai Du contributed to the conception of this study and revised the manuscript.

\section{Acknowledgments}

We are extremely grateful to Wuhan Health Bureau, and all the hospitals and community health centers involved in this study. 


\section{Abbreviations}

BMI: body mass index; PTB: preterm birth; GWG: gestational weight gain; IOM: Institute of Medicine

\section{References}

1. Goldenberg RL, Culhane JF, lams JD, Romero R. Epidemiology and causes of preterm birth. The lancet. 2008;371(9606):75-84.

2. Katz J, Lee AC, Kozuki N, Lawn JE, Cousens S, Blencowe H, et al. Mortality risk in preterm and smallfor-gestational-age infants in low-income and middle-income countries: a pooled country analysis. The Lancet. 2013;382(9890):417-25.

3. Mwaniki MK, Atieno M, Lawn JE, Newton CR. Long-term neurodevelopmental outcomes after intrauterine and neonatal insults: a systematic review. The Lancet. 2012;379(9814):445-52.

4. Robbins CL, Hutchings Y, Dietz PM, Kuklina EV, Callaghan WM. History of preterm birth and subsequent cardiovascular disease: a systematic review. American journal of obstetrics and gynecology. 2014;210(4):285-97.

5. Blencowe H, Cousens S, Oestergaard MZ, Chou D, Moller A-B, Narwal R, et al. National, regional, and worldwide estimates of preterm birth rates in the year 2010 with time trends since 1990 for selected countries: a systematic analysis and implications. The Lancet. 2012;379(9832):2162-72.

6. Cnattingius S, Villamor E, Johansson S, Bonamy A-KE, Persson M, Wikström A-K, et al. Maternal obesity and risk of preterm delivery. Jama. 2013;309(22):2362-70.

7. Shaw GM, Wise PH, Mayo J, Carmichael SL, Ley C, Lyell DJ, et al. Maternal prepregnancy body mass index and risk of spontaneous preterm birth. Paediatric and perinatal epidemiology. 2014;28(4):30211.

8. Mamun AA, Callaway LK, O'Callaghan MJ, Williams GM, Najman JM, Alati R, et al. Associations of maternal pre-pregnancy obesity and excess pregnancy weight gains with adverse pregnancy outcomes and length of hospital stay. BMC pregnancy and childbirth. 2011;11(1):1.

9. Faucher M, Hastings-Tolsma M, Song J, Willoughby D, Gerding Bader S. Gestational weight gain and preterm birth in obese women: a systematic review and meta-analysis. BJOG: An International Journal of Obstetrics \& Gynaecology. 2016;123(2):199-206.

10. Sharma AJ, Vesco KK, Bulkley J, Callaghan WM, Bruce FC, Staab J, et al. Associations of gestational weight gain with preterm birth among underweight and normal weight women. Maternal and child health journal. 2015;19(9):2066-73.

11. Yaktine AL, Rasmussen KM. Weight Gain During Pregnancy:: Reexamining the Guidelines: National Academies Press; 2009.

12. Laitinen J, Jääskeläinen $A$, Hartikainen $A L$, Sovio U, Vääräsmäki M, Pouta $A$, et al. Maternal weight gain during the first half of pregnancy and offspring obesity at 16 years: a prospective cohort study. BJOG: An International Journal of Obstetrics \& Gynaecology. 2012;119(6):716-23. 
13. Swank ML, Caughey AB, Farinelli CK, Main EK, Melsop KA, Gilbert WM, et al. The impact of change in pregnancy body mass index on the development of gestational hypertensive disorders. Journal of perinatology : official journal of the California Perinatal Association. 2014;34(3):181-5.

14. Bei-Fan Z. Predictive values of body mass index and waist circumference for risk factors of certain related diseases in Chinese adults: study on optimal cut-off points of body mass index and waist circumference in Chinese adults. Asia Pacific journal of clinical nutrition. 2002;11(s8):S685-S93.

15. Macdonald-Wallis C, Tilling K, Fraser A, Nelson SM, Lawlor DA. Gestational weight gain as a risk factor for hypertensive disorders of pregnancy. Am J Obstet Gynecol. 2013;209(4):327 e1-17.

16. Pantham $P$, Aye I, Powell T. Inflammation in maternal obesity and gestational diabetes mellitus. Placenta. 2015;36(7):709-15.

17. Lee HJ, Ha JE, Bae KH. Synergistic effect of maternal obesity and periodontitis on preterm birth in women with preeclampsia: a prospective study. Journal of clinical periodontology. 2016.

18. Khatibi A, Brantsaeter A-L, Sengpiel V, Kacerovsky M, Magnus P, Morken N-H, et al. Prepregnancy maternal body mass index and preterm delivery. American journal of obstetrics and gynecology. 2012;207(3):212. e1-. e7.

19. Shin D, Song WO. Prepregnancy body mass index is an independent risk factor for gestational hypertension, gestational diabetes, preterm labor, and small-and large-for-gestational-age infants. The Journal of Maternal-Fetal \& Neonatal Medicine. 2015;28(14):1679-86.

20. Parker MG, Ouyang F, Pearson C, Gillman MW, Belfort MB, Hong X, et al. Prepregnancy body mass index and risk of preterm birth: association heterogeneity by preterm subgroups. BMC pregnancy and childbirth. 2014;14(1):1.

21. Goldstein, R. F. , et al. "Association of Gestational Weight Gain With Maternal and Infant Outcomes: A Systematic Review and Meta-Analysis." Obstetric Anesthesia Digest 38.2(2018):64-65.

22. McDonald SD, Han Z, Mulla S, Lutsiv O, Lee T, Beyene J, et al. High gestational weight gain and the risk of preterm birth and low birth weight: a systematic review and meta-analysis. Journal of Obstetrics and Gynaecology Canada. 2011;33(12):1223-33.

23. Huber LRB. Validity of self-reported height and weight in women of reproductive age. Maternal and child health journal. 2007;11(2):137-44.

24. Johnson J, Clifton RG, Roberts JM, Myatt L, Hauth JC, Spong CY, et al. Pregnancy outcomes with weight gain above or below the 2009 Institute of Medicine guidelines. Obstetrics and gynecology. 2013;121(5):969.

\section{Supplementary Files}

This is a list of supplementary files associated with this preprint. Click to download.

- SuppData.docx

- additionalfile2.docx 
- additionalfile1.docx

Page 15/15 